\title{
ILCEA
}

Revue de l'Institut des langues et cultures

d'Europe, Amérique, Afrique, Asie et Australie

21 | 2015

Discours politique et culturel dans la Russie

contemporaine

\section{La notion d'État en russe moderne à travers les associations verbales}

The Notion of State in Modern Russian: Word Associations

\section{Valentina Toujikova}

\section{OpenEdition}

Journals

Édition électronique

URL : http://journals.openedition.org/ilcea/3025

DOI : 10.4000/ilcea.3025

ISSN : 2101-0609

Éditeur

UGA Éditions/Université Grenoble Alpes

Édition imprimée

ISBN : 978-2-84310-294-3

ISSN : 1639-6073

Référence électronique

Valentina Toujikova, "La notion d'État en russe moderne à travers les associations verbales ", ILCEA

[En ligne], 21 | 2015, mis en ligne le 01 février 2015, consulté le 01 mai 2019. URL : http://

journals.openedition.org/ilcea/3025; DOI : 10.4000/ilcea.3025

Ce document a été généré automatiquement le 1 mai 2019.

(C) ILCEA 


\section{La notion d'État en russe moderne à travers les associations verbales}

The Notion of State in Modern Russian: Word Associations

Valentina Toujikova

1 La notion d'État en Russie attire aujourd'hui l'attention des linguistes, des historiens et des spécialistes en droit. Certes, cette notion a toujours été au cœur des débats, mais ces deux dernières décennies marquent un intérêt croissant pour les notions politiques et sociales. Plusieurs chercheurs français et russes comme Valéry Kossov (2002), Jurij Karaulov, Olga Evtušenko et Igor' Ružickij (2006) ont consacré leurs travaux à l'étude du concept d'État en russe moderne.

2 Dans le présent article, nous nous intéresserons à la perception de la notion d'État à travers les associations citées dans les dictionnaires des associations verbales du russe. L'objectif de cet article est d'analyser les associations liées au mot gosudarstvo qui mettent en évidence les spécificités de la perception de cette notion dans la vie quotidienne. Dans une perspective diachronique, nous nous servirons des résultats mentionnés par le public de différents âges pour attester de l'évolution du comportement associatif.

3 Nous attirerons également l'attention sur le caractère paradigmatique et syntagmatique de diverses associations. En effet, nous verrons que certaines associations témoignent d'une synonymie ou antonymie partielle ou peuvent entrer dans des relations métaphoriques, métonymiques et former des expressions de types variés. De plus, nous utiliserons les citations tirées de la presse russe pour observer la corrélation entre les réponses des Russes mentionnées dans les dictionnaires et les associations employées par les journalistes.

4 Avant de procéder à l'analyse des résultats, nous jugeons nécessaire de présenter quelques points théoriques concernant l'association verbale et son rôle dans les études sémantiques, ainsi que de décrire la constitution de notre corpus. 


\section{Association verbale et constitution du corpus}

5 Les recherches sur l'association verbale prennent leurs racines dans la psychologie expérimentale. François Jodelet distingue trois stades de leur évolution (1965 : 94). Si le premier stade, appelé "mentaliste", étudie l'association des mots pour décrire les fondements de la pensée humaine, le second privilégie la description des liaisons associatives verbales dans les thermes de théories « stimulus - réponse », se focalisant sur les différences individuelles. Enfin, le troisième stade dépasse le cadre behavioriste et conduit à l'étude de la structure générale des liaisons associatives verbales.

Quelques dates concernant les recherches des psychologues et des linguistes peuvent tracer l'apparition des dictionnaires des associations verbales. En 1901, les chercheurs allemands en psychologie, Albert Thumb et Karl Marbe (1901), ont utilisé l'expérience associative pour l'étude du comportement langagier. Les expériences des psychologues allemands se sont basées sur le test associatif, qui consistait à prononcer des noms de parenté devant plusieurs personnes en leur demandant de répondre par d'autres mots. Les chercheurs ont obtenu des noms de parenté en tant qu'associations, par exemple père-mère. Cette expérience associative $a$ été introduite ensuite dans la linguistique et, en particulier, dans les études sémantiques. En 1910, la première liste des associations verbales a été publiée par Grace Helen Kent et Aaron Rosanoff (1910 : 37-96, 317-390), qui ont rassemblé les 100 mots les plus fréquents dans la vie quotidienne selon les réactions de 1000 personnes interrogées. L'apparition de cette liste a été suivie par la publication en $1973 \mathrm{du}$ premier dictionnaire An associative thesaurus of English and its computer analysis (Thésaurus associatif de l'anglais et son analyse par ordinateur), dirigé par George Kiss (Kiss, Armstrong, Milroy \& Piper, 1973 : 153-165) et par celle en 1977 du dictionnaire des normes associatives de la langue russe Slovar'associativnyh norm russkogo jazyka de Leontiev (1977). Se basant sur les travaux d'Aleksej Leontiev, les linguistes russes Jurij Karaulov, Evgenij Tarasov et Natalija Ufimceva ont crée plusieurs dictionnaires des associations verbales du russe, de l'espagnol, du biélorusse, du bulgare et autres (Sančes, Karaulov \& Čerkasova, 2001; Ufimceva, Čerkasova, Karaulov \& Tarasov, 2004). Ces dictionnaires, contrairement à leurs homologues dits traditionnels, ne présentent pas de définitions mais des champs associatifs créés à la base des mots cités par les locuteurs non spécialistes en linguistique. Selon Michèle Debrenne, Claude Frey et Mary-Annick Morel, ces dictionnaires permettent de décrire «les connaissances qu'une société donnée attache à un certain mot à une période donnée de son histoire » (2008:1118).

7 En effet, le mot évoque chez l'individu non seulement un concept, mais un ensemble de connaissances, « un ensemble de représentations, de sentiments, d'attitudes " (Caron, 2008: 86). Il est question de connotations qui peuvent être individuelles, mais aussi communes à un groupe d'individus et notre but est de dégager les connotations typiques pour la perception du mot gosudarsrvto chez les Russes. Comme l'a affirmé François Jodelet, l'association verbale dépend de plusieurs variables : de personnalité, de situation et également culturelles (1965: 117-123). Dans notre étude, nous analyserons la variété des associations en fonction de l'âge. Notre corpus est constitué de deux sources : Russkij associativnyj tezaurus (Thesaurus associatif russe) et Russkij associativnyj slovar: associativnye reakcii škol'nikov I-XI klassov (Dictionnaire associatif russe : les associations des élèves du primaire et du secondaire). Pour le premier dictionnaire, le test associatif effectué par des chercheurs russes a englobé des personnes entre 10 et 100 ans, sur une 
durée de dix ans de 1987 à 1997. Il convient de préciser qu'il existe deux versions de dictionnaire : Russkij associativnyj tezaurus (Thesaurus associatif russe) en version en ligne et Russkij associativnyj slovar' (Dictionnaire associatif russe) en version papier. L'avantage de la version en ligne donne la possibilité de comparer les résultats selon l'âge, la profession et le sexe, contrairement à la version papier. Principalement, les personnes interviewées sont des étudiants de différentes spécialités de la $1^{\mathrm{re}}$ à la $3^{\mathrm{e}}$ année de Licence, âgés de 17 à 25 ans, de différentes régions de Russie, pour qui le russe est la langue maternelle. Nous avons retrouvé 73 réactions pour le mot gosudarstvo proposées par les personnes interrogées ayant plus de 25 ans.

$8 \quad$ Le Dictionnaire associatif russe: les associations des élèves du primaire et du secondaire a réuni les réponses des élèves de la région de Saratov sur la période de 1998 à 2008. De plus, les auteurs ont distingué les réponses selon l'âge des élèves. À notre avis, la comparaison des réactions proposées par les personnes de différents âges mettra en évidence les spécificités de la perception de la notion d'État par ces dernières.

Enfin, une autre question est liée à la détermination du caractère des liens entre le stimulus et ses réactions. En étudiant l'association verbale et les liaisons associatives, Jodelet a choisi la classification proposée par Karwoski et Berthold, qui distinguent 6 catégories logiques et 2 catégories non logiques : «1) Similitude essentielle (synonymie, analogie) : grand-large, rugueux-rêche ; 2) Identification générale ("superordination " de Wells) ; 3) Identification spécifique (subordination: la classe de $\mathrm{S}$ inclut celle de R) : légume-chou ; 4) Identification contingente (proximité spatiale ou relations fonctionnelles en S et R); œuf-breakfast... ; 5) Opposition essentielle («contraste » de Wells) ; 6) Opposition contingente (coordination : $\mathrm{S}$ et $\mathrm{R}$ appartiennent à une même classe $\mathrm{C})$ : pied-main...; 7) Définition opérationnelle ( $\mathrm{R}$ implique une action sur ou un mouvement vers S): plancher-laver; 8) Complètement de phrase (restitution d'une locution): chou-fleur...» (Jodelet, 1965: 109-110). François Jodelet a signalé l'inconvénient de cette classification qui ne tient pas compte de la nature grammaticale de $\mathrm{S}$ et de $\mathrm{R}$. Nous verrons plus loin dans l'analyse des réponses obtenues que les associations de type nom-adjectif ou nom-verbe sont répandues. Nous partageons l'opinion de François Jodelet quand il dit que la classification de Karwoski et Schlacter comporte quelques catégories incertaines, ce qui a été corrigé plus tard par ces chercheurs par la réduction du nombre de catégories. Ainsi, nous distinguerons des réponses appartenant à la catégorie d'opposition (d'après la définition de Wells il est question de la catégorie de contraste) et celle d'équivalence.

Pour le Russkij associativnyj slovar' nous préférons la traduction: Dictionnaire des associations verbales $d u$ russe, car cette traduction nous semble plus appropriée dans un contexte où la notion d'association est ambiguë. La définition de l'association comme «action de réunir des éléments divers pour former un ensemble» ou comme "fait psychologique par lequel les représentations et les concepts sont susceptibles de s'évoquer mutuellement" (Le Nouveau Petit Robert: 157) peut renvoyer aux différents types d'associations, tandis que la notion de l'association verbale est plus concrète dans le contexte psycholinguistique. François Jodelet a insisté sur le fait que « tous les mots qu'un parleur est capable de proférer distinctivement, soit de façon isolée, soit successivement au sein d'un discours suivi » (1965: 95) présentent un système d'unités du comportement verbal. Il appelle ce système « celui d'opérateurs verbaux disponibles » qui comporte un certain nombre de liaisons associatives que nous avons déjà mentionné plus haut. 


\section{Analyse des résultats}

11 Les résultats obtenus dans les deux dictionnaires sont présentés selon le type de recherche: recherche directe et recherche inverse. La base des données directe comprend les réactions au stimulus réparties dans l'ordre décroissant de fréquence. La statistique indique le nombre total de réactions obtenues, de réactions différentes, d'absentions et de réactions uniques. Dans la base des données inverse, l'article comporte plusieurs stimuli. Les chiffres indiquent le nombre de fois où cette réaction a été évoquée en réponse. Dans notre article, nous nous limiterons aux résultats de la base directe, qui nous renseigne d'une façon abondante sur les liens sémantiques les plus probables entre les mots.

Dans le Russkij associativnyj tezaurus le stimulus gosudarstvo a obtenu 645 réactions au total, parmi lesquelles 237 réactions différentes, 157 uniques et 6 abstentions. Parmi toutes ces réactions, les trois premières selon l'ordre de fréquence sont: strana (pays) avec 49 réponses, naše (notre) avec 26 réponses et vlast' (pouvoir) avec 20 réponses; l'âge des personnes interrogées étant de 10 à 100 ans. Si nous limitons notre recherche aux résultats obtenus avec des personnes âgées entre 25 et 100 ans, nous obtenons des données différentes: le stimulus gosudarstvo a obtenu 86 réactions au total, parmi lesquelles 59 réactions différentes, 46 uniques et 1 abstention; les trois premières sont pravovoe (de droit) avec 7 réponses, naše (notre), Sovetskoe (soviétique) et strana (pays) avec 5 réponses. Il faut tenir compte du fait que les personnes ayant plus de 25 ans étaient moins nombreuses lors de cet interview.

En nous référant aux résultats du Russkij associativnyj slovar': associativnye reakcii škol'nikov I - XI klassov (2011 : 88), nous constatons que la plupart des élèves identifient le mot gosudarstvo à leur propre pays. La réaction Rossija avec 28 réponses (I-IV), 29 (V-VI) et 17 réponses (VII-VIII) prédomine et cède la place au mot strana seulement dans les réponses des élèves d'IX-XI classes.

Dans l'ensemble, nous retrouvons plusieurs réactions communes aux deux dictionnaires, mais avec une fréquence différente : strana (pays), pravo (droit), vlast' (pouvoir), mašina (machine), carstvo (royaume), prezident (président), gorod (ville), Rossija (la Russie), bol'šoe (grand), demokratii ou demokratija (de démocratie ou démocratie), pravitel'stvo (gouvernement), plohoe (mauvais)... En revanche, les associations proposées par les personnes de plus de 25 ans dans le Russkij associativnyj tezaurus reflètent plus de spécificités, par exemple les réactions Rossija ou vlast' sont absentes.

Une autre spécificité est reflétée par les réactions renvoyant au passé soviétique. L'expression Sovetskoe gosudarstvo (État soviétique) démontre que les gens âgés de plus de 25 ans et qui ont vécu pendant la période soviétique identifient facilement le mot gosudarstvo au mot soviétique. L'ancrage de l'époque soviétique est encore tenace, car même dans les réponses des Russes de 17 à 25 ans nous retrouvons les réactions socialističeskoe (socialiste) avec 17 réponses, SSSR (Union des républiques socialistes soviétiques) avec 15 réponses, sovetskoe (soviétique) avec 14 réponses, i revolucija (et révolution) avec 12 réponses, Sovetov (des Soviets) avec 4 réponses. Il semblerait que les changements politiques parviennent à effacer les réactions renvoyant au passé soviétique, car nous ne retrouvons qu'une seule réaction SSSR(1) dans le Russkij associativnyj slovar': associativnye reakcii škol'nikov I - XI klassov. De plus, il s'agit des lycéens 
des trois dernières classes. Dans les réponses des élèves plus jeunes, il n'y a plus aucune référence au passé soviétique.

Nous avons retrouvé quelques réactions reflétant le caractère démocratique de l'État dans les deux dictionnaires: pravovoe/de droit (18 réponses), svobodnoe/libre (5 réponses), demokratii/de démocratie (2 réponses), demokratičeskoe/démocratique (2 réponses), zakon/ loi (1 réponse) et golosovat'/voter (1 réponse), parlament/parlement (1 réponse), deputat/ député (1 réponse), demokratija/démocratie, Duma/Douma (8).

Si l'association Duma ${ }^{1}$ renvoie à la réalité russe, les associations pravovoe (de droit), svobodnoe (libre), demokratija (démocratie) peuvent concerner n'importe quel État : réel ou hypothétique. Serait-il question de la considération par les Russes de l'État actuel en tant qu'État de droit, libre et démocratique ? Ou peut-être, s'agit-il de l'image qu'ils se font de n'importe quel État? Ou encore, ces associations prennent-elles leurs racines dans les expériences individuelles et sont-elles liées aux changements politiques en Russie ? La présence du pronom naše (notre) dans les résultats des personnes interrogées avant 1997, ainsi que la réaction Rossija parmi les réponses des élèves interviewés récemment, permet de supposer que les Russes associent avant tout le mot gosudarstvo à l'État russe. Comme nous l'avons mentionné dans notre thèse, la notion d'État de droit a commencé à pénétrer dans le discours politique en 1985, avec la perestroïka initiée par Mikhaïl Gorbatchev pour objectif de former un État de droit socialiste (Toujikova, 2012: 198-199). Selon les réponses obtenues dans les deux dictionnaires, nous pouvons admettre que les Russes espèrent que leur État sera un État de droit.

Dans la presse russe, nous avons retrouvé plusieurs fois l'expression à caractère métaphorique stroit' pravovoe gosudarstvo (construire un État de droit) :

ДЕМОНТИРОВАВ ТОТАЛИТАРНУЮ СИСТЕМУ, МЫ ВПЕРВЫЕ В СВОЕЙ ИСТОРИИ НАЧАЛИ

СТРОИТЬ ДЕМОКРАТИЧЕСКОЕ ОБЩЕСТВО И ПРАВОВОЕ ГОСУДАРСТВО. (Izvestija, 2002)

«En démontant le système totalitaire, nous avons commencé pour la première fois

dans notre histoire à construire une société démocratique et un État de droit. »

Cette métaphore de construction, très répandue dans la presse russe ${ }^{2}$, indique qu'il est question de la phase de construction et non de l'aboutissement d'un processus; pour d'autres, l'État de droit est une fiction :

ХОРОШО ИНОГДА ПОМЕЧТАТЬ И ПРЕДСТАВИТЬ НЕ СЕРУЮ РОССИЙСКУЮ ДЕЙСТВИТЕЛЬНОСТЬ,

А, К ПРИМЕРУ, НАСТОЯЩЕЕ ПРАВОВОЕ ГОСУДАРСТВО... ПОНЯТНОЕ ДЕЛО, ЧТО НАШЕ ГОСУДАРСТВО ЯВЛЯЕТСЯ ПРАВОВЫМ ЧИСТО НОМИНАЛЬНО. (Moskovskij komsomolec, 2013)

« Il est parfois bien de rêver et d'imaginer non pas une réalité russe médiocre, mais par exemple un véritable État de droit... Il est clair que notre État apparaît comme un État de droit simplement par le nom. »

19 En revenant à la comparaison des réactions, nous remarquons la diminution des réponses pour le mot strana (pays) chez les personnes âgées de plus de 25 ans. Si nous considérons que la réaction strana appartient à la relation d'équivalence, nous constatons un changement dans les stéréotypes linguistiques, où cette relation évolue et devient plus importante chez les jeunes contrairement à la génération précédente.

Un autre phénomène souligné dans les travaux de Jurij Karaulov, ainsi que dans notre thèse, est lié à la connotation souvent défavorable des réactions au stimulus gosudarstvo. Ainsi, si nous prenons seulement les réponses des Russes de plus de 25 ans, nous rencontrons les réactions non répétitives comme : dragon (dragon), drjan' (saleté), durakov (d'imbéciles), mašina (machine, mécanisme), plohoe (mauvais), rabov (d'esclaves), idiotov (d'idiots) (version en ligne du Thesaurus associatif russe). Le même phénomène est manifeste dans les réponses des élèves de différents âges : plohoe (mauvais), vory (voleurs), 
duraki (imbêciles), gruzit' (barber), zloe (méchant), mašina (machine, mécanisme), obnanglelo (il a eu le culot), stremnoe (malsain)... (Russkij associativnyj slovar', 2011 : 88). Les mots à valeur péjorative comme gruzit', stremnoe, provenant du langage familier, caractérisent la spécificité des réponses données par les jeunes des dernières années.

La réaction drakon (dragon) au stimulus gosudarstvo rappelle l'emploi fréquent de la métaphore gosudarstvo-sverkh"estestvennoe suščestvo (l'État est une créature surnaturelle) dans le discours médiatique d'aujourd'hui. Dans notre thèse, nous avons analysé plusieurs images de ce type de métaphore qu'Anatolij Baranov et Jurij Karaulov citent dans leur dictionnaire des métaphores politiques russes : gosudarstvo-monstr (l'État est un monstre), gosudarstvo-drakon (l'État est un dragon), gosudarstvo-cerber (l'État est un cerbère) (Baranov \& Karaulov, 1994 : 107-108).

L'association gosudarstvo-drakon (l'État est un dragon) renvoie à la Bible où le monstre marin sous le nom hébreu de Léviathan est représenté sous différentes formes : dragon, serpent ou crocodile. Thomas Hobbes décrit l'État sous ce nom, pour souligner sa puissance. Dans la mythologie ancienne des slaves du Sud, le serpent avec des ailes contribua à la fertilité des terres et protégea les récoltes grâce à ses pouvoirs sur l'eau ( $\mathrm{L} a$ mythologie slave : le dictionnaire encyclopédique, 2011 : 185). En revanche, les slaves de l'Est et de l'Ouest le considéraient comme un démon, séduisant et tuant les femmes. En effet, dans les légendes, dans les bylines et dans les contes russes, le dragon à plusieurs têtes, dont le nombre peut varier de 3 à 12 , représentait le mal. Vladimir Propp, folkloriste russe, consacra tout un chapitre à cette créature mythique dans son œuvre Les Racines historiques du conte merveilleux. Selon lui, ce dragon appelé « zmej» (serpent) peut habiter dans l'air, dans l'eau ou dans les montagnes (Zmej Gorynyč). Cette créature peut cracher le feu et voler de belles jeunes femmes, de préférence de jeunes princesses : «Zmej unosit carevnu na svoih ognennyh kryljah.» («Le dragon emporte la princesse sur ses ailes de feu. » Propp, 2009 : 183-207)

Plus tard, l'association du dragon aux peuples nomades s'installa chez les Russes qui en avaient subi les nombreuses invasions, occupations ou simples razzias. Après la conquête de Kazan', le dragon apparait dans le blason de cette ville. Selon Nazym Hanzafarov (2001), la croyance que les Tatares vénéraient le dragon ou le serpent appelé Zilant est fausse. S'appuyant sur les sources de l'historien Mihail Hudrjakov, Nazym Hanzafarov souligne que «les musulmans associaient le serpent au paganisme et les chrétiens à l'islam » (2001: 69). C'est pourquoi il est plus probable que le dragon, présent dans le blazon de Kazan', fut introduit par les Russes pour glorifier leur victoire sur les Tatares.

24 À l'époque soviétique avec l'installation du régime totalitaire, l'image «l'État est un dragon" continue à susciter une attitude défavorable. Dans son article " "Drakon" E. Švarca i "Železnaja misterija” D. Andreeva: uslovnye modeli dramy v literature 19401959 godov $^{3}$ »(2008), Olga Daševskaja a étudié le pouvoir et ses mécanismes dans l'État totalitaire, symbolisé par l'empire du dragon. Ce symbolisme permet à Švarc et à Andreev d'interpréter et d'installer une perception négative de l'État totalitaire chez les lecteurs. Aujourd'hui, les journalistes utilisent souvent cette métaphore "l'État est un dragon " pour désigner les États asiatiques comme la Chine, le Japon ou la Corée :

«ДАЛЬНЕВОСТОЧНЫЕ ДРАКОНЫ» ПОСЕТИЛИ ФУКУСИМУ. (Nezavicimaja gazeta, 2011)

«"Les dragons de l'Extrême-Orient" ont visité Fukusima. »

АЗИАТСКИЕ «ТИГРЫ» И «ДРАКОНЫ» ГОТОВЫ ПЕРЕГРЫЗТЬ ДРУГ ДРУЖКЕ ГОРЛО. (VO

Geopolitika, 2014) 
"Les "tigres" et "dragons" asiatiques sont prêts à s'égorger l'un l'autre. »

Dans le deuxième extrait, nous rencontrons également la métaphore animalière qui transmet une attitude négative envers la politique des États asiatiques, car il s'agit de la conduite agressive des animaux qui veulent «s'égorger l'un l'autre». Nous partageons ainsi l'opinion de plusieurs linguistes comme Eduard Budaev, Galina Skljarevskaja ou Anatolij Čudinov, qui affirment que « dans le discours politique le recours à la source du monde animalier sert à créer chez le lecteur l'image négative de la cible » (Budaev, 2007 : 86).

La réaction mašina est relative à la métaphore mécanique, très répandue dans la presse russe actuelle et largement étudiée par les linguistes français et russes, comme Vladimir Beliakov (2006), Anatolij Baranov et Jurij Karaulov (1997). Selon leur opinion, ainsi que d'après les citations retrouvées dans la presse, cette métaphore est dotée d'une connotation péjorative.

Voici un exemple tiré d'un article du Corpus de la langue russe :

ГОСУДАРСТВЕННАЯ МАШИНА СИЛЬНО ОТСТАЛА ОТ СТРАНЫ И ЯВЛЯЕТСЯ ТОРМОЗОМ НА ПУТИ ПРОГРЕССА. (Nezavisimaja gazeta, 2003)

«La machine étatique s'est fortement laissée dépasser par le pays et freine le progrès. "

Il est important de souligner que l'auteur distingue ici l'État et le pays, ce qui correspond aux résultats obtenus auprès des Russes ayant plus de 25 ans, attestant la faible fréquence de l'association entre ces mots.

Les réactions durakov (d'imbéciles) et rabov (d'esclaves) peuvent former les expressions gosudarstvo durakov (État d'imbéciles) et gosudarstvo rabov (État d'esclaves). L'association durakov provient de l'expression strana durakov (pays d'imbéciles), utilisée dans l'adaptation russe de Pinocchio, La Petite Clé d'or ou Les Aventures de Bouratino, publiée en 1936 par Aleksej Tolstoj. Dans ce conte, Bouratino, marionnette, fabriquée par le père Karlo, rencontre deux escrocs, le renard Alice et le chat Bazilio, qui lui proposent de l'amener au pays des imbéciles pour visiter le fameux champ de miracles où, selon eux, on peut semer l'argent et obtenir un vrai arbre avec des billets au lieu de feuilles. Dans la presse russe actuelle, les journalistes ont souvent recours à ces expressions :

ПОЛЕ ПЕНСИОННЫХ ЧУДЕС В СТРАНЕ ДУРАКОВ. (Gazeta39, 2012)

"Le champ des miracles pour les retraites se trouve au pays des imbéciles. »

НАША СТРАНА - ЭТО ГОСУДАРСТВО РАБОВ: ЧЕМ БЕДНЕЕ ЧЕЛОВЕК, ТЕМ БОЛЬШЕ ОН ДОЛЖЕН

ГОСУДАРСТВУ. (Investstorm, 2013)

« Notre pays c'est un État d'esclaves : plus l'homme est pauvre, plus il est redevable

à l'État. »

Dans ces exemples, nous observons de l'ironie et de l'autocritique et s'il est question d'un État d'imbéciles et d'esclaves, comment appeler ses citoyens?

Enfin, l'absence de la réaction vlast' au stimulus gosudastvo dans les réponses des personnes âgées de plus de 25 ans et sa forte présence ( 20 réponses) chez les personnes de 10 à 25 ans ont attiré notre attention dans la comparaison des résultats. Dans notre thèse, nous avons montré que, d'après son étymologie, le mot gosudarstvo, apparu au $\mathrm{xv}^{\mathrm{e}}$ siècle et signifiant «akt pravlenija» (action de gouverner), «gosudarstvovanie » (règne), comporte l'actant du pouvoir dominant qui renvoie à l'autorité d'une seule personne, du monarque, d'où vient la personnification de vlast'. Nous avons également mis en évidence l'association du mot vlast' aux mots gosudarstvo, pravitel'stvo (gouvernement) qui se remarque souvent dans le langage courant et nous en avons cité plusieurs exemples tirés de la presse russe actuelle. Nous croyons que l'emploi courant de cette association par les 
mass médias, ces dernières années, a amplifié chez les jeunes Russes l'identification entre vlast' et gosudarstvo, contrairement aux Russes plus âgés qui ont une tendance à les distinguer.

La différence des résultats dans le Russkij associativnyj slovar': associativnye reakcii škol'nikov I-XI klassov consiste également en la présence de noms propres d'hommes politiques dans les réponses des élèves du collège et du lycée, comme Poutine (I-XI classes) avec 3 réponses, Elcin (V-VI) et Žirinovskij (IX-XI) avec une seule réponse. Ces dernières années, les hommes politiques, en particulier les chefs d'État et les membres du gouvernement, participent activement aux rencontres avec les jeunes en Russie. Ainsi, ces derniers sont inévitablement plus impliqués dans la vie politique de l'État que l'était la génération précédente.

\section{Comparaison des résultats avec d'autres langues}

Dans notre thèse, après avoir analysé les résultats pour les mots gosudarstvo/état dans les dictionnaires des associations verbales du russe, du français et de l'anglais, nous avons révélé quelques associations communes avec des fréquences différentes telles que strana (49) / pays (99) / country (9), pravitel'stvo (5) / gouvernement (16) / government (4)... ainsi qu'un nombre important d'associations spécifiques (Toujikova, 2012: 276-279). Nous avons soulevé le problème de polysémie du mot État en français et du mot state en anglais pour notre analyse des réactions. Si les deux premières réactions pays (99) / nation (31) et country (9) / America (6) nous renvoient au terme appartenant au vocabulaire politique et social, les troisièmes santé (20) / condition (4) sont liées au sens premier : manière d'être (physique, intellectuelle, morale) d'un être vivant. Cependant, il est clair que le sens relevant du domaine politique prédomine avec davantage de réponses.

31 Nous observons une différence dans les réactions françaises, anglaises et russes de type noms géographiques et noms propres, l'absence de réactions marquant l'aspect dimensionnel dans les réponses chez les Français et les anglophones contrairement à ceux donnés par les Russes : bol'šoe/grand (16); velikoe/grand, ogromnoe/immense (10)... Enfin, un grand nombre de réactions transmettant une appréciation favorable, par exemple bogatoe/riche (6), mogučee/puissant, fort, svobodnoe/libre (5), sil'noe/fort, spravedlovie/juste (3), moguščestvennoe/puissant, fort ou défavorable comme razval/ effondrement (3), $v$ upadke/être en déclin (2), bespravnoe/privé de droits, besčelovečnoe/ inhumain, drjannoe/mauvais, gniloe/pourri, zastojnoe/stagnant, ploxoe/mauvais (1), témoignent d'une grande importance de l'affectif chez les Russes.

Il est intéressant de noter qu'il existe plus de réponses communes données par les Russes et les Français vlast' (21) / pouvoir (3), pravo (18) / droit (16). Ceci permet d'affirmer que la perception de la notion d'État chez les Français et les Russes contient plus de ressemblances comparées à celle du monde anglophone. Malgré l'universalisme du concept, l'analyse des résultats pour les mots État, gosudarstvo et state reflète, de façon cohérente, les particularités du russe dans la perception de la notion d'État. Une analyse plus détaillée des réactions aux mots du vocabulaire politique et social pour les langues différentes pourrait s'imposer dans des travaux de recherches ultérieurs. 


\section{Conclusion}

Les résultats de cette étude permettent de dégager quelques caractéristiques relatives à la perception de la notion d'État dans le russe moderne. La tendance générale des deux comportements, du public adolescent et adulte, met en évidence la perception négative de cette notion par le biais d'associations à valeur péjorative. Si plusieurs personnes interrogées avant 1997 donnent encore des associations favorables - velikoe (grand) avec 10 réponses, bogatoe (riche), mogučee (puissant, fort) avec 6 réponses, svobodnoe (libre), rodnoe (natal) avec 5 réponses, sil'noe (fort), spravedlivoe (juste) avec 3 réponses, moguščestvennoe (puissant) avec 2 réponses (Russkij associativnyj tezaurus, version en ligne) -, les associations favorables sont uniques chez les élèves du primaire et du secondaire : ser'eznoe (sérieux), bogatoe (riche), krutoe (nickel), krepkoe (solide) (Russkij associativnyj slovar', $2011: 88$ ).

En comparant les réponses proposées par les personnes de différents âges, nous avons observé une influence des variables sur l'association verbale. Le contexte vécu ressort fortement dans cette comparaison et on assiste, au sein d'une langue unique, à une évolution des stéréotypes linguistiques, d'une génération à l'autre. Le passé soviétique s'efface et cède la place aux nouvelles réalités politiques et sociales. Le nombre d'associations à valeur favorable et dimensionnelle voit son déclin. L'association entre l'État et le nom géographique d'appartenance s'accroît. Le champ associatif évolue en fonction des nouvelles connaissances de la vie politique, mais retient toujours certaines connaissances accumulées par les générations précédentes: comme strana (pays), pravitel'stvo (gouvernement), prezident (président), pravo (droit) et d'autres.

\section{BIBLIOGRAPHIE}

BARANOV Anatolij \& KARAULOV Jurij (1994), Slovar' russkix političeskix metaphor, Moscou : Institut russkogo jazyka AN SSSR.

BELIAKOV Vladimir (2006), « La réalité russe à travers la métaphorisation des discours médiatiques ", Metaphorik.de, 10.

BUDAEv Eduard (2007), Postsovetskaja dejstvitel'nost' v metaforah rossijskoi i britanskoj pressy, Nižnij Tagil : Nižnitagil'skaja gosudarstvenno-social'naja pedagogičeskaja akademija.

CARON Jean (2008), Précis de psycholinguistique, Paris : Presses universitaires de France.

ČUVAKIN Oleg (2014), « Aziatskie “tigry” i “drakony” gotovy peregryzt' drug družke gorlo », VO Geopolitika, publié le 24 juillet, <http://topwar.ru/54869-aziatskie-tigry-i-drakony-gotovyperegryzt-drug-druzhke-gorlo.html> (1er août 2014).

DAŠEVSKAJA Olga (2008), « “Drakon” E. Švarca i “Železnaja misterija” D. Andreeva: uslovnye modeli dramy v literature 1940-1959 godov », Vestnik TGPU, 2, <http://cyberleninka.ru/article/n/ 
drakon-e-shvartsa-i-zheleznaya-misteriya-d-andreeva-uslovnye-modeli-dramy-v-

literature-1940-h-1950-h-godov> (18 janvier 2014).

DEBRENNE Michèle, FREY Claude \& MOREL Mary-Annick (2008), « L'étude des champs associatifs du français : création d'un dictionnaire des normes associatives ", Congrès mondial de linguistique française, <http://dx.doi.org/10.1051/cmlf08131> (12 novembre 2009).

GRIGOR'EV Andrej (2013), « Komensacija za VSM. V Borovom Matjušino iskat' ne probovali? », Moskovskij komsomolec, publié le 25 décembre, <http://kazan.mk.ru/article/2013/12/25/964304kompensatsiya-za-vsm-v-borovom-matyushino-iskat-ne-probovali.html> (3 janvier 2014).

HANCEvič Olesja (2011), « Dal'nevostočnye drakony posetili Fukusimu », Nezavicimaja gazeta, publié le 23 mai, <www.ng.ru/world/2011-05-23/8_fukusima.html> ( $1^{\text {er }}$ août 2014).

HANZAFAROV Nazym (2001), Simvoly Tatarstana (mify i real'nost'), Kazan' : Fiker.

JODELET François (1965), « L'association verbale », Traité de psychologie expérimentale (93-137), Paris : Presses universitaire de France.

KARAULOV Jurij, EVTUŠENKo Olga \& RUŽICKIJ Igor' (dir.) (2006), Russkoe slovo v russkom mire - 2005: Gosudarstvo i gosudarstvennost'v jazykovom soznanii rossijan, Moscou : Azbukovnik.

KARAULOV Jurij, SOROKIN Jurij, TARASOv Evgenij, UfIMCEVA Nadežda \& ČERKASOVA Galina (dir.) (2002) , Russkij associativnyj slovar'. Kniga 1. Prjamoj slovar': ot stimula k reakcii. Associativnyj tezaurus sovremennogo russkogo jazyka, Moscou : Izdatel'stvo Astrel'.

KENT George \& ROSANOFF Aaron Joshua (1910), « A Study of Association in Insanity », American Journal of Insanity, 67(1-2), 37-96, 317-390.

Kossov Valéry (2002), «Culture politique et traduction. Le concept de l'État en français et en russe », ILCEA, 3, <http://ilcea.revues.org/index773.html> (21 décembre 2013).

LEONTIEV Aleksei (1977), Slovar' associativnyh norm russkogo jazyka, Moscou : Izdatel'stvo Moskovskogo unviersiteta.

Le nouveau Petit Robert (2000), Josette Ray-Debove et Alain Rey (dir.), Paris : Le Robert.

MERZLJAKOV Stas (2012), « Pole pensionnyh čudes v strane durakov », Gazeta39, <http:// gazeta39.ru/kld/people-n-events/558-pens.html> (5 janvier 2014).

NAZARBAEv Nursultan (2002), «Legkikh del v politike ne byvaet », Izvestija, publié le 3 avril, < http://izvestia.ru/news/260299> (3 janvier 2014).

NEMCov Boris (2003), « Strategija Rossii », Nezavisimaja gazeta, publié le 27 mai, < www.ruscorpora.ru> (4 janvier 2014).

PROPP Vladimir (2009), Istoričeskie korni volšebnoj skazki, Moscou : Izdatel'stvo Labirint.

Russkij associativnyj slovar': associativnye reakcii škol'nikov I - XI klassov (2011), t. 1, Saratov : Izdatel'stvo Saratovskogo universiteta.

Russkij associativnyj tezaurus (Thesaurus associatif russe), <http://tesaurus.ru/dict/dict.php>. SANČES Puig Marija, KARAUlov Jurij \& ČERKASOVA Galina (2001), Associativnye normy ispanskogo $i$ russkogo jazykov, Moscou-Madrid : Azbukovnik.

Slavjanskaja mifologija. Enceklopedičeskij slovar' (2011), Moscou : Meždunarodnye otnošenija. THUMB Albert \& MARBE Karl (1901), Experimentelle Untersuchungen über die psychologischen Grundlagen der sprachlichen Analogiebildung, Leipzig : Engelmann. 
Toujiкоva Valentina (2012), L'analyse lexico-sémantique comparative des mots et de la notion "État/ gosudarstvo" dans les langues française et russe, thèse dirigée par Antoine Nivière, Université Nancy 2.

Ufimceva Nadežda, čERKASOva Galina, KARAulov Jurij \& TARAsov Evgenij (dir.) (2004), Slavjanskij associativnij slovar': russkij, belorusskij, bolgarskij, ukrainskij.

\section{NOTES}

1. La Douma d'État est la chambre du parlement bicaméral russe.

2. Dans son article "La réalité russe à travers la métaphorisation des discours médiatiques ", Vladimir Beliakov souligne également l'emploi courant de la métaphore du bâtiment dans la presse, qui sert à révéler le caractère instable de la société russe. Il mentionne l'extrait d'un article dans lequel la Russie est comparée au chantier.

3. " "Dragon" de E. Švarc et "Mystère de fer" de D. Andreev : modèles conventionnels de drame dans la littérature des années 1940-1950».

\section{RÉSUMÉS}

L'objectif de cet article consiste à démontrer la perception des Russes à propos de la notion d'État dans leur vie quotidienne. Pour cela, nous procédons à l'analyse des associations liées au mot gosudarstvo dans les dictionnaires des associations verbales du russe. Nous observons ici le caractère de certaines liaisons associatives et comparons les réponses des personnes en fonction de leur âge, ce qui permettra de dégager l'évolution des stéréotypes linguistiques dans le comportement verbal, d'une génération à l'autre. De plus, nous avons recours à la presse russe actuelle pour établir la corrélation entre les associations mentionnées dans les dictionnaires et leur emploi dans le discours.

The objective of this article is to demonstrate the Russian perception of the notion of State in everyday life. In order to do this, we are going to analyze the words that were produced in association to the stimulus gosudarstvo in Russian associative dictionaries. We are examining here the character of some associative relations and comparing the answers of subjects in relation of age that allow us to get an evolution of linguistic stereotypes, from one generation to the next. Furthermore, we are referring to actual Russian newspapers with a view to establish a correlation between associations in dictionaries and their use in discourse.

\section{INDEX}

Mots-clés : État, association verbale, comportement verbal, stéréotype, discours

Keywords : State, word association, verbal behavior, stereotype, discourse 


\section{AUTEUR}

\section{VALENTINA TOUJIKOVA}

Docteur ès Langues, Littératures et civilisations, Laboratoire CERCLE (EA 7342), Université de Lorraine 